\title{
Combining Location2Vec with LSTM to predict next locations from trajectory data
}

Shanshan HAN ${ }^{\text {a }}$, Fu REN ${ }^{\text {a,b }}$ and Qingyun DU ${ }^{\text {a,b,c,d* }}$

${ }^{a}$ School of Resource and Environmental Sciences, Wuhan University, Wuhan, China, Shanshan HAN, hanshanshan@whu.edu.cn, Fu REN,renfu@whu.edu.cn, QingyunDU,qydu@whu.edu.cn

${ }^{b}$ Key Laboratory of Geographic Information Systems, Ministry of Education, Wuhan University, Wuhan, China;

${ }^{c}$ Key Laboratory of Digital Mapping and Land Information Application Engineering, National Administration of Surveying, Mapping and Geoinformation, Wuhan University, Wuhan, China;

${ }^{d}$ Collaborative Innovation Center of Geospatial Technology, Wuhan University, Wuhan, China

* Corresponding author

Keywords: trajectory data; LSTM; Location2Vec; location prediction

\begin{abstract}
:
Research on human location prediction is beneficial personalized location recommendations, location-based advertising, and ride-sharing services. The prediction of future locations is generally based on historical trajectory data. With the development and prevalence of mobile sensors, large volumes of data that can capture human trajectories are generated and provide researchers with more adequate and accurate data to study human trajectories and location predictions. In this paper, we propose a model containing long short-term memory (LSTM) to understand historical trajectories and predict future locations. LSTM is a special recurrent neural network (RNN) architecture that contains three gates and one internal state to control the storage of information. Additionally, we leverage the LINE (Large-scale Information Network Embedding), an embedding method to transform vertices into large scale networks, to transform locations into spatial vectors according to their distance information, which we name it "Location2Vec". By feeding the summation of the dynamic location embeddings and pre-trained Location2Vec embeddings into LSTM model, it can effectively predict next locations of a trajectory and improve the predicting accuracy. Finally, we verify the reliability of the model, and the results show that our model outperforms other traditional trajectory models.
\end{abstract}

